\title{
PeerVote: A Decentralized Voting Mechanism for P2P Collaboration Systems
}

\author{
Thomas Bocek, Dalibor Peric, Fabio Hecht, David Hausheer, \\ and Burkhard Stiller \\ Department of Informatics IFI, University of Zurich, Switzerland \\ \{bocek, peric, hecht, hausheer, stiller\}@ifi.uzh.ch
}

\begin{abstract}
Peer-to-peer (P2P) systems achieve scalability, fault tolerance, and load balancing with a low-cost infrastructure, characteristics from which collaboration systems, such as Wikipedia, can benefit. A major challenge in $\mathrm{P} 2 \mathrm{P}$ collaboration systems is to maintain article quality after each modification in the presence of malicious peers. A way of achieving this goal is to allow modifications to take effect only if a majority of previous editors approve the changes through voting. The absence of a central authority makes voting a challenge in $\mathrm{P} 2 \mathrm{P}$ systems.

This paper proposes the fully decentralized voting mechanism PeerVote, which enables users to vote on modifications in articles in a P2P collaboration system. Simulations and experiments show the scalability and robustness of PeerVote, even in the presence of malicious peers.
\end{abstract}

\section{Introduction}

Peer-to-peer (P2P) systems inherently support redundancy, scalability, fault tolerance, and load balancing 2016] at a lower cost than client/server systems, since every participating user contributes with resources. These advantages incited the emergence of different P2P-based applications, including audio and video streaming [28, file-sharing [7], and storage [24 27]. A large-scale collaboration system, such as Wikipedia [26], could also benefit from the aforementioned characteristics of $\mathrm{P} 2 \mathrm{P}$ systems [3]. In a $\mathrm{P} 2 \mathrm{P}$ collaboration system, users share the resources necessary to host and distribute articles that can be modified by any other user.

A major challenge in $\mathrm{P} 2 \mathrm{P}$ collaboration systems is to assure that usergenerated content quality is being maintained or improved after each modification, despite the lack of a central authority. Since article quality is a highly subjective measurement, user-based voting is needed to allow users to express their opinion on whether or not a proposed modification shall be accepted.

The contribution of this paper is PeerVote, a decentralized voting mechanism that provides a method to maintain quality of content and its modifications. The proposed voting mechanism ensures that every modification to a document has the approval from the majority of previous editors. This helps to prevent vandalism, editing wars, and deliberate censorship. To the best of our knowledge, this is the first user-based voting scheme for P2P collaboration systems. PeerVote 
has been implemented in a $\mathrm{P} 2 \mathrm{P}$ collaboration application and evaluated on top of a structured P2P network using a simulator and EMANICSLab [10. PeerVote can be used for any kind of document writing with a primary focus on distributed collaboration, such as scientific reports, project deliverables, or online articles.

The remainder of this paper is structured as follows. While Section 2 discusses related work, Section 3 shows the design of PeerVote. In Section 4 implementation details and evaluation results are presented. Finally, Section 5 concludes this paper and suggests future work.

\section{Related Work}

One way of achieving the goal of improving quality of content in P2P collaboration systems is by using recommendation or reputation systems. 1] proposes a content-driven reputation system for Wikipedia, which is based on automatic analysis of edit-changes of Wikipedia articles and side-steps any user-based rating. The deriving author reputation can be used to predict the quality of future articles of such authors. Korsgaard and Jensen [14] outline the integration of a recommendation system in Wikipedia, which allows users to express their preference about the article and consult its general score, whereas the resulting system would still remain centralized. Although such systems can be used in conjunction with PeerVote, their focus is on the document itself, while PeerVote's main concern is on document's update compliance. While several P2P Wiki approaches exist $18 / 25 / 1723$, none of these approaches support user-based voting.

Different voting and consensus reaching algorithms have been proposed, with distributed systems and electronic voting as their main applications. In distributed systems, the main purpose of voting mechanisms is to ensure consistency among replicated data, usually by achieving an agreement between replica holder entities. Examples of such consensus protocols are the two-phase commit protocol [12, the weighted voting [11, and the decentralized weighted voting [19. With the exception of the latter, such voting schemes have the disadvantage of being centralized. Another disadvantage of those schemes is that they are synchronous, thus, their application for human-based voting would require all voters to express their preference simultaneously, which is unfeasible for large numbers of participants.

Fully decentralized voting protocols, such as the inexact voting over wide area networks [13] and the Deno voting protocol [5] have been proposed. The first approach shows a message complexity quadratic with respect to the number of voters, which is not scalable. The Deno voting protocol, while being scalable, does not guarantee that updates commit in a bounded time.

Secret ballot protocols, or electronic voting protocols, implement a democratic voting system on electronic equipment. Some existing implementations 621] address specific security concerns, like eligibility, privacy, individual and universal verifiability, fairness, robustness, and receipt-freeness, but they work in a centralized fashion.

Table 1 shows a comparison among these voting mechanisms. None of these mechanisms reviewed support a decentralized user-based voting. 
Table 1. Comparison of distributed voting mechanisms

\begin{tabular}{|l||c|c|c|c|c|}
\hline & $\begin{array}{l}\text { Decen- } \\
\text { tralized }\end{array}$ & Scalable & $\begin{array}{l}\text { Time- } \\
\text { bounded }\end{array}$ & $\begin{array}{l}\text { Asyn- } \\
\text { chronous }\end{array}$ & User-based \\
\hline 2-Phase Commit & No & Yes & Yes & No & No \\
\hline Weighted Voting & No & Yes & Yes & No & No \\
\hline $\begin{array}{l}\text { Decentralized } \\
\text { Weighted Voting }\end{array}$ & Yes & Yes & Yes & No & No \\
\hline Secure Ballot & No & Yes & Yes & Yes & Yes \\
\hline Inexact Voting & Yes & No & Yes & Yes & No \\
\hline Deno Voting & Yes & Yes & Yes & No & No \\
\hline PeerVote & Yes & Yes & Yes & Yes & Yes \\
\hline
\end{tabular}

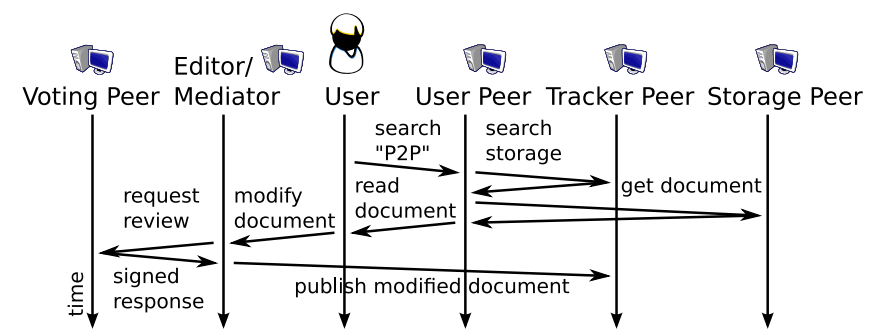

Fig. 1. PeerVote message sequence chart

\section{PeerVote Design}

The design of PeerVote defines roles and their interactions. Each role defines a set of actions and interacts with one or more other roles. The following use case introduces these roles.

\subsection{Use Case}

A typical use case for PeerVote is shown in Figure1, where a user searches for a document including the keyword "P2P". The peer finds a tracker with addresses of peers holding documents with the keyword "P2P". After reading, the user finds a wrong statement and corrects it. Following the majority of previous authors agreeing on this correction, the modified document is published.

\subsection{Roles}

PeerVote defines six roles: tracker, storage, user, editor, mediator, and voter roles (cf. Figure 1). One peer can have one or more roles, depending on the action of a user. 
Tracker Role: A tracker peer stores references to storage peers that store certain documents. Each tracker peer is responsible for storing references to documents with document-ids nearest to its peer-id. A tracker peer also evaluates voting metadata. A tracker can reject references with wrong or inconsistent voting metadata. References have a time-to-live value, so they are removed in case peers stop (re)publishing them.

Storage Role: A storage peer stores and periodically (re)publishes documents. Publishing is carried out by searching for trackers responsible for a particular document-id and storing addresses of storage peers and metadata. These documents are stored by peers that either created or viewed them.

User Role: A user interacts via a user interface with its user peer. The role of the user peer is to search for, download, and display documents. A search is carried out by searching trackers responsible for a particular document-id. These trackers reply with references to storage peers from which a user peer can download this document. If user peers publish downloaded documents, they also become storage peers.

Editor Role: An editor peer proposes modifications to a document. The editor hands over the change proposal to the mediator peer, which initiates the voting session.

Mediator Role: A mediator peer is responsible for a voting session. After a modification to a document is proposed, the mediator contacts its voting peers and requests them to review and sign the result. If a majority of those voters approve the change, the modified document is published. In the publishing process, references and the voting metadata are stored on tracker peers.

Voter Role: A voter peer can vote exactly once on a modification proposal. Allowed voters are editors of previous modifications of this document or editors with many approved votes.

\subsection{Voting Algorithm and Data Structure}

A voting session is initiated when an editor submits a proposal and becomes a mediator. As a mediator, it starts the voting phase by sending the proposal to all previous editors or to editors with many edits, if no previous editors exist in case of a new document. These editors can vote, if the change shall be accepted. The voting session is open for a specific amount of time. During this time, voters can review and vote for or against the change. If a voter does not vote, it is considered as a negative vote. Thus, there are no benefits of avoiding to contact peers for a review. A vote is accepted, if signed voting results reach a threshold. Figure 2 shows the pseudo code for the voting scheme, with the methods voting() and incoming (). The voting method is used by a mediator to start a voting session, while the incoming method is called on voting peers (previous editors).

In a decentralized system, it is important to take measures against dropping votes, i.e., when a mediator ignores negative votes. For example, if a mediator 


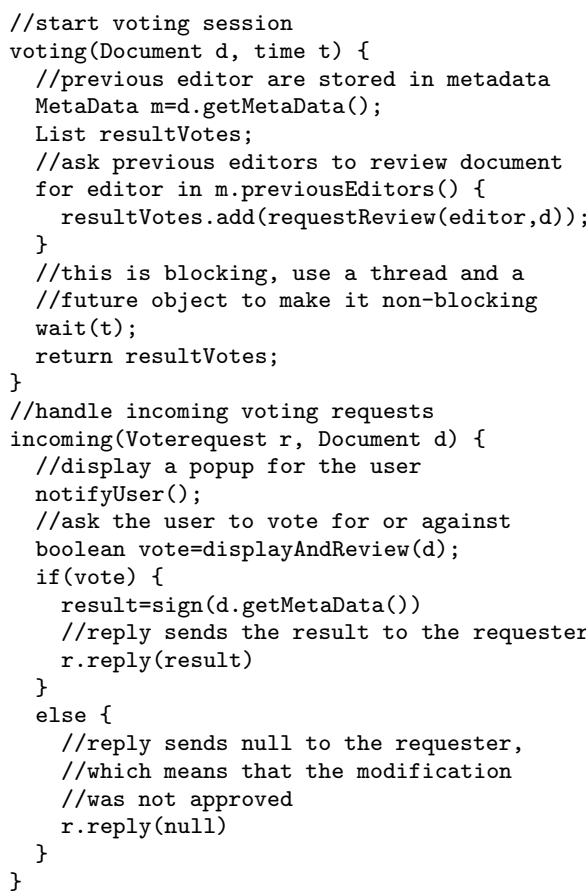

Fig. 2. Voting scheme pseudo code

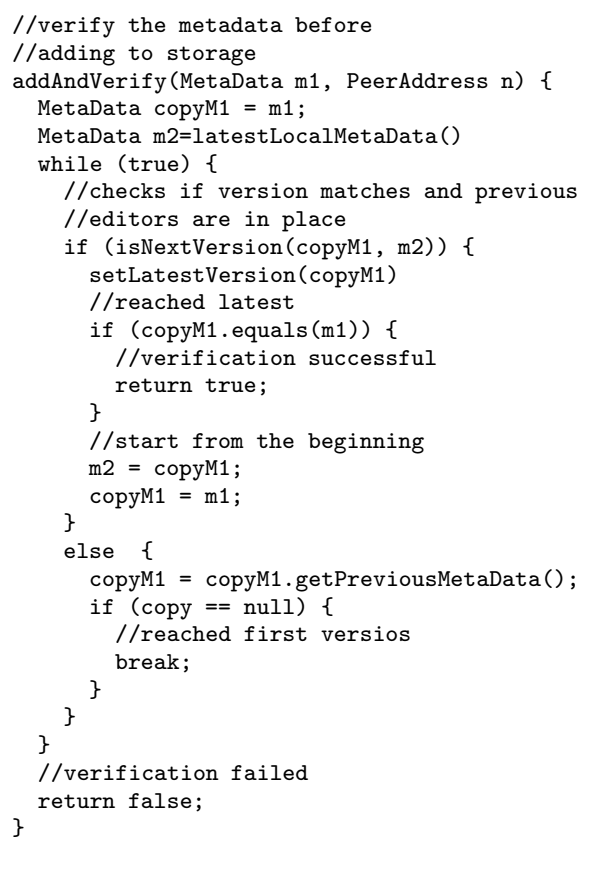

Fig. 3. Voting metadata verification

receives 98 negative votes and 2 positive votes, dropping 97 negative votes gets the modification accepted. One way of dealing with this is to introduce a threshold for a minimum number of votes for a valid outcome. Another way is to count no votes as negative votes and use a threshold for the positive votes to determine the voting outcome. PeerVote uses the second approach, thus dropping negative votes does not change the voting outcome.

Storage and user peers need to evaluate the voting metadata to detect unreviewed changes. The information required for this evaluation is stored in the voting metadata. The voting metadata in PeerVote consists of versioning information, hash code of the content of the document, document-id, and signed voting results. The document-id is constant for all changes on the same base document. The voting metadata has the structure as shown in Table 2

It is important to verify the consistency of previous voting sessions. Otherwise, a malicious peer could replace, add, or drop signatures to pretend to have less editors or to be a previous editor. Thus, voting metadata is verified with the

Table 2. Voting metadata structure and example values

\begin{tabular}{|l|l|l|l|l|l|}
\hline $\mathrm{Nr}$ & Version & Document-id & Hash & Signatures $($ Signature, Peer-id) & Previous \\
\hline \hline$\# 1$ & 1 & $0 \mathrm{x} 123$ & $0 \mathrm{x} 234$ & $(0 \mathrm{x} 134,0 \mathrm{x} 456)$ & \\
\hline$\# 2$ & 2 & $0 \mathrm{x} 123$ & $0 \mathrm{x} 235$ & $(0 \mathrm{x} 135,0 \mathrm{x} 456)(0 \mathrm{x} 136,0 \mathrm{x} 567)$ & $\# 1$ \\
\hline
\end{tabular}


following rules. Each version can have at most one new editor, a new version contains all peer-ids of previous editors, and all versions have the signed results of the voting sessions. These rules are verified as shown in Figure 3, which shows the pseudo code for verification of voting metadata. The voting scheme uses public-key cryptography for peer identification and signatures to prevent forgery. A public key is exchanged on first contact.

In such a decentralized system, peers can modify documents concurrently, because they operate independently. Concurrency in this voting scheme for change proposals uses a first come, first served scheme. This means that for conflicting changes, the peer that collected the votes first, publishes the change proposal and all other conflicting change proposals with the same version number are discarded. The user which submitted the failed change proposal is notified and can update to the latest version of the document and propose the change again.

\subsection{Voting Scheme Example}

The following example explains the design and the pseudocode, presented in Figure 3 with example values. While the new document $(\# 1)$ in Table 2 with document-id 0x123 and hash value 0x234 has no reference to previous metadata, the modified document $(\# 2)$ has a reference to $(\# 1)$, which means that (\#2) is based on (\#1). For the modified document (\#2), the hash value $0 \times 235$ changed, because of the modified content. The signature in the new document (\#1) is based on the hash value 0x234 to confirm that its content was approved by peer with peer-id 0x456. This signature belongs to peer 0x456 in this example. All signatures of the modified document (\#2) show that two voters have approved this modification, the original editor, and a second editor with peer-id 0x567. The signature for document \#2 is based on the hash $0 \times 235$.

\section{Implementation and Evaluation}

PeerVote has been implemented in a $\mathrm{P} 2 \mathrm{P}$ collaboration application, which has the primary focus on evaluating generic decentralized collaboration. Thus, the simulation does not focus on parameters for specific applications such as Wikipedia. This P2P collaboration application is based on TomP2P 2], a Distributed Hash Table (DHT) and tracker implementation.

\subsection{P2P Collaboration Application Implementation}

The P2P collaboration application supports document publishing, document downloading, and document modifying using a tracker-based approach. Publishing a new document requires first to search for a tracker with a peer-id closest to the document-id. A tracker is responsible for storing voting metadata and document references to storage peers. For new documents, the voting metadata is filled and stored with the reference to the storage peer on the tracker. A new document is accepted without any further validation because it is assumed that a new document is never published by a malicious peer. 
Downloading a document starts with searching for trackers with a peer-id close to the document-id. These trackers contain addresses of peers that store the document. After those trackers are found, addresses and voting metadata are downloaded. The addresses from the trackers are used to download the document.

After a document is modified, all previous editors are requested to review the change. All voting peers that are online answer this request as described in the incoming method in the pseudo code of Figure 2. If a sufficient number of voters have signed the modified document, the document is published. For publishing a modified document, the tracker verifies voting metadata as described in the addAndVerify method (cf. Figure 3). If the verification is successful, the reference to the modified document and voting metadata is stored. Further search requests to this tracker return the modified metadata and its reference to the storage peer.

\subsection{Simulation and Experimental Settings}

Simulations and experiments have been run to investigate scalability, faulttolerance, and robustness. This has been performed by using various combinations of the following parameters: number of peers, number of malicious peers, number of voting sessions, and number of change proposals. Since user-based voting in $\mathrm{P} 2 \mathrm{P}$ collaboration applications have not been studied before, parameters, such as churn (10\%), concurrency (50\%), and number of publishing peers $(10 \%$, $20 \%, 30 \%$ ), have been selected. Malicious peers can propose incorrect modifications, vote against correct modifications, or vote randomly. Malicious peers that vote randomly simulate users that vote without reviewing, because users may pretend to be active without investing resources for reviewing. A random voting peer votes with a probability of $50 \%$ for and with a probability of $50 \%$ against it. Malicious peers that propose incorrect modifications simulate users that intend to publish unsuitable information, e.g., spam or contradicting information. Malicious peers that vote against correct proposals simulate colluding peers. A malicious vote is always detected because malicious modifications are marked as such and considering different opinions on modifications is not supported. In the current setting, the voting metadata is not verified by user peers, because malicious storage peers are not implemented. New documents do not have wrong information regardless of the publishing peer being malicious. A voting session lasts at most 45 seconds and a peer replies, if online, within 10 seconds to finish the simulation and experiments in a reasonable time. The voting meta data is accumulated during these changes. There is a $50 \%$ chance for peers to propose changes concurrently in a voting session. Currently, in the P2P collaboration application changes based on the same version are always conflicting and never merged.

Signatures are required to sign the result of a voting session. The current implementation generates a hash of the modified data as a replacement for the signature, because signing and verifying is CPU intensive and many peers are simulated on one machine, which makes the CPU a bottleneck. Since malicious 
peers do not exploit this, a signature can be verified by hashing the modified data. Thus, a distribution of public keys is not required.

PeerVote's evaluations are based on more than 1,000 peers. Churn has been set to $10 \%$, which means that peers fail to reply in $10 \%$ of the time. Experiments and simulations have been run 10 times each and the averages have been calculated including the standard deviation.

All simulations have been performed on $2 \operatorname{Intel}(\mathrm{R}) \mathrm{Xeon}(\mathrm{R})$ quad-core CPUs, $2.83 \mathrm{GHz}$, with a Java HotSpot(TM) 64-Bit Server VM (build 11.2-b01, mixed mode). The parameters set for the simulation with Java were -d64 -Xmx10G, which allows to run in 64bit mode and use 10 GB RAM. The simulation required 2 days to complete. All experiments were run on 14 EMANICSLab 10 machines. EMANICSLab is a research network established among European research partners and consists of 7 different partner sites. The hardware used in EMANICSLab is heterogeneous with different CPU models and RAM sizes. The experiment required 2.5 days to complete.

\subsection{Results and Discussion}

Figure 4 shows the accumulated number of DHT and voting messages per peer with an increasing number of peers, from 100 to 1000 . The number of published documents is set to $10 \%, 20 \%$, and $30 \%$ respectively, proportional to the number of peers, which means that for $20 \%$ and 100 peers, 20 documents are published, for 1000 peers, 200 documents are published. A document is changed 2 times. All change proposals are carried out by $30 \%$ of the peers. This means that for 100 peers, 30 change proposals are submitted for 20 documents. Thus, some proposals are submitted concurrently. Malicious peers are not present in this simulation. Figure 5 shows the accumulated number of voting messages per peer with increasing number of peers using the aforementioned parameters.

Figure 4 indicates a logarithmic behavior for all three curves. Furthermore, Figure 5 shows a constant number of messages from 100 to 1,000 peers, and that the number of voting messages doubles if the publishing peers double. This indicates that the voting algorithm is scalable with an increasing number of peers. While the voting messages have a small number of messages (maximum of 4.75), the overall number of messages, including DHT overhead have a maximum of 575. Thus, this indicates that the DHT overhead contributes most to the number of messages.

Figure 6] shows the graph for voting traffic with an increasing number of documents, from $10 \%$ to $100 \%$ proportional to the number of peers. The number of peers shown are 400,500, and 600. Voting parameters are set as described in the previous simulation. Malicious peers are not present. Figure 7 shows a graph for voting traffic with an increasing number of change proposals with the same settings as for Figure 6.

Both figures show for 400, 500, and 600 peers, that the 3 traffic graphs overlap. This indicates that the voting traffic is independent of the number of peers. The voting traffic is dependent on the number of documents (Figure 6) and the number of change proposals (Figure 7). 


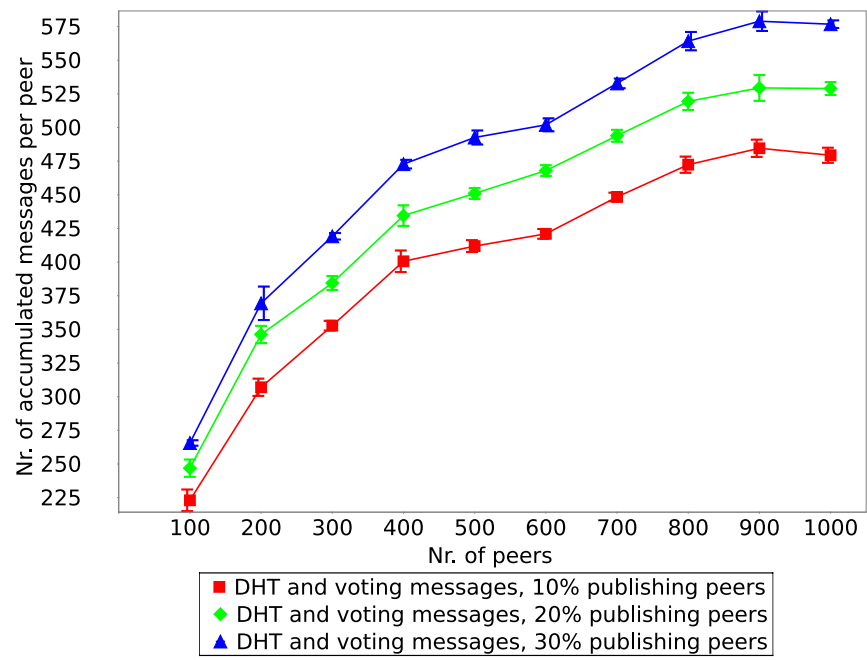

Fig. 4. Number of voting and DHT messages per peer

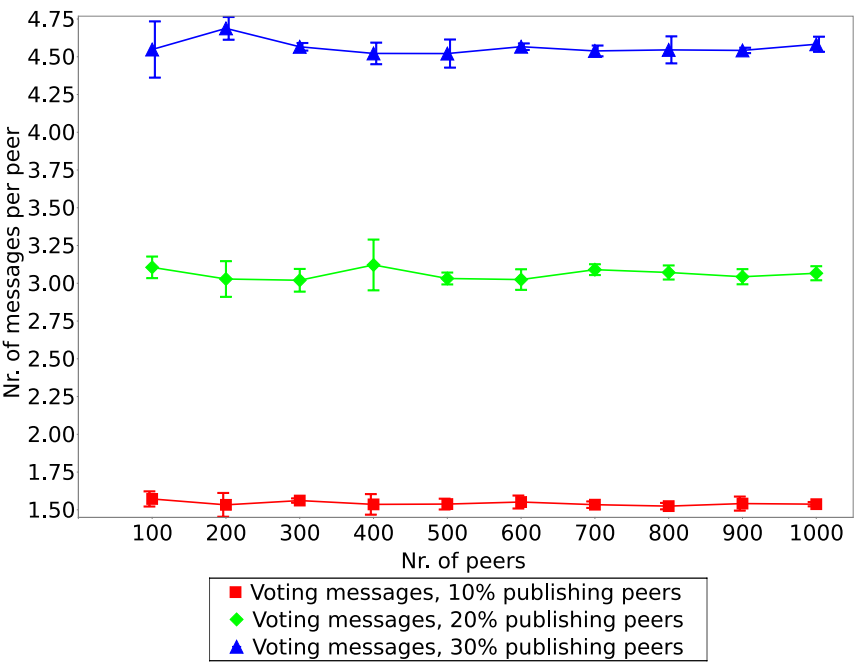

Fig. 5. Number of voting messages per peer

Figure 6 shows that traffic increases with linear complexity for an increasing number of peers proposing a change, while Figure 7 shows that traffic increases with polynomial complexity for an increasing number of change proposals per peer. The graph in Figure 7 is explained due to the increasing number of previous authors, which increase the message size and also the number of messages, because all previous peers need to be contacted. Thus, the list of previous authors for a document needs to have a fixed capacity. 


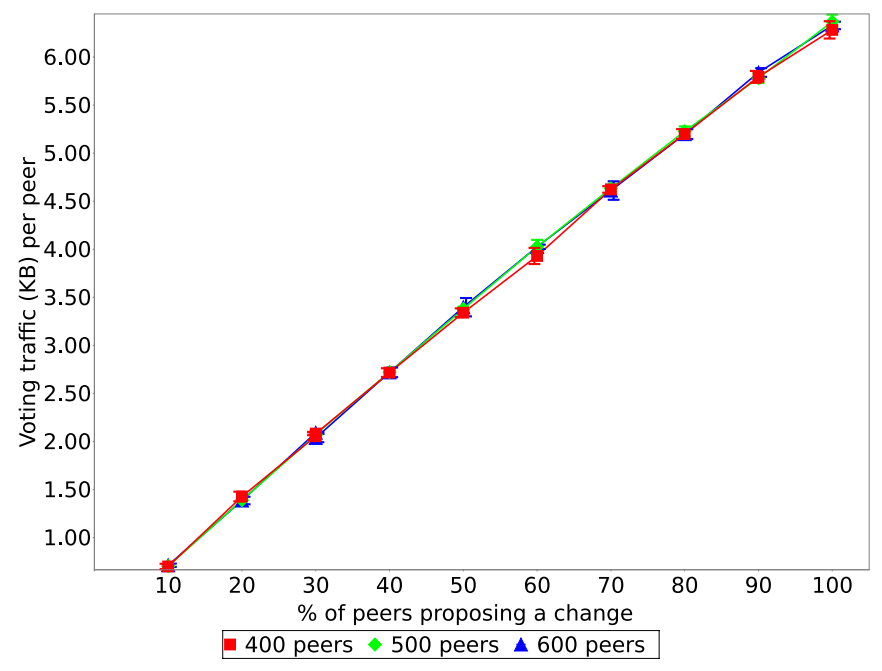

Fig. 6. Voting traffic for increasing number of proposing peers

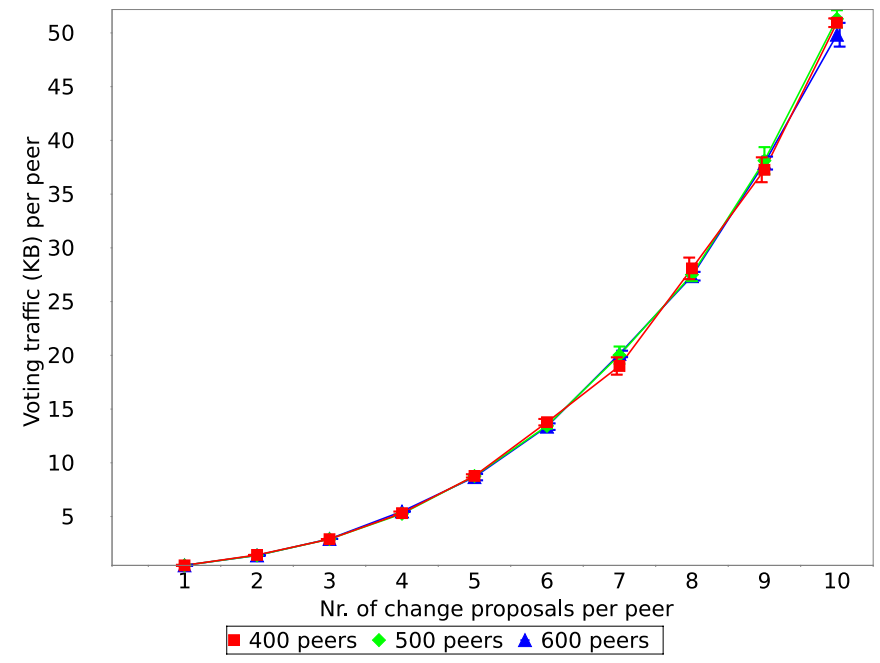

Fig. 7. Voting traffic for increasing change proposals per peer

To evaluate malicious behavior, up to $50 \%$ malicious peers have been simulated. Figure 8 shows an increasing number of malicious peers. The number of peers are set to 500. The number of published documents is set to 100. Both graphs are decreasing and start at $100 \%$ correct documents. The more malicious peers join, the more incorrect changes are present. The graph with random votes (Figure 8) has less incorrect changes compared to the graph with malicious votes. For $50 \%$ of malicious peers (250 peers), $65 \%$ correct documents are stored, while for $50 \%$ of random voting peers, $82 \%$ correct documents are stored. More than 


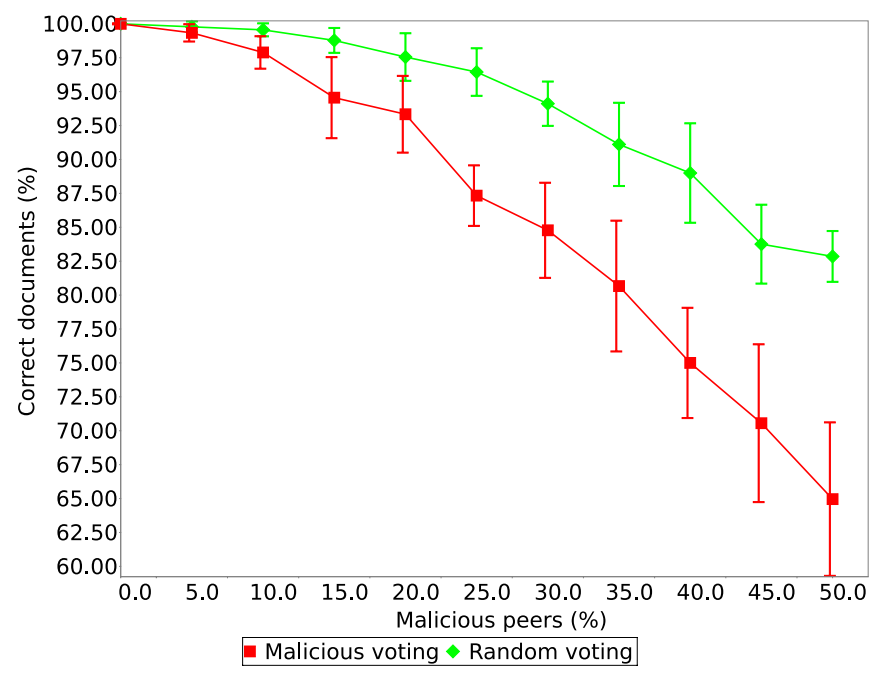

Fig. 8. Effect on documents with malicious and randomly voting peers

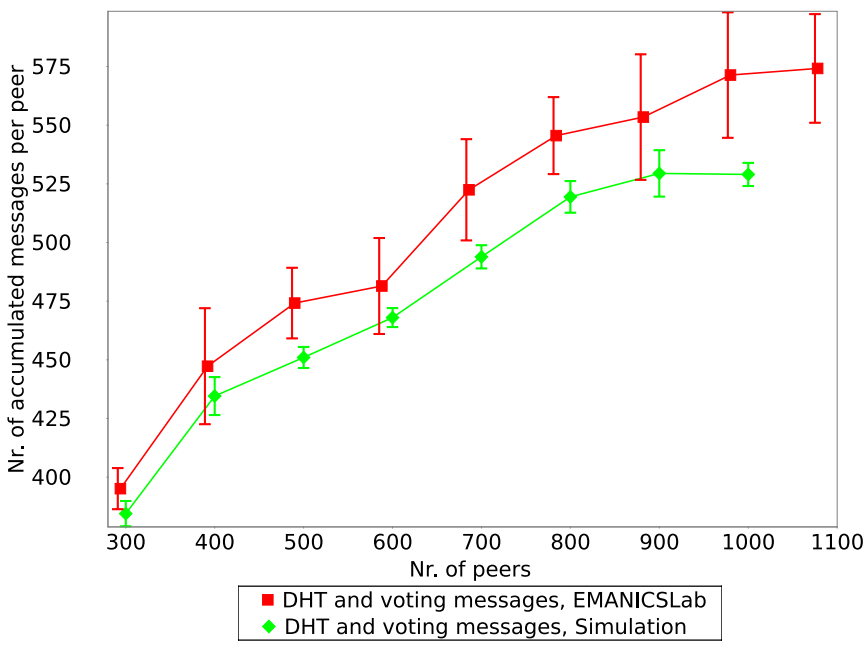

Fig. 9. Experiment on EMANICSLab and simulation comparison

half of the documents are correct, because the initial document is always correct, regardless if a peer is malicious or not.

Figure 9 shows the comparison of those simulation results above with the experimental results from the EMANICSLab implementation. Both settings have from 300 to less than 1,100 peers and 20\% published documents. Two changes are proposed and the graph shows the number of DHT and voting messages.

The number of messages for the DHT and voting messages is higher on EMANICSLab, which is also observed for other comparisons. The higher message 
number is due to peers on EMANICSLab, which may fail to reply or which send a reply too late, because of other CPU or network intensive experiments running on EMANICSLab nodes. The larger error bars for EMANICSLab experiments also reflect this.

\section{Summary, Conclusions, and Future Work}

This paper presented PeerVote, a decentralized voting mechanism in a P2P collaboration application. Experiments and simulations with a prototypical implementation showed that PeerVote is scalable and robust, even in the presence of random and malicious voting peers. Evaluations showed that overall traffic increases logarithmically and that voting messages are independent on the number of peers. Furthermore, experiments on EMANICSLab showed that the algorithm deployed in a real network has similar traffic characteristics as in the simulation.

PeerVote considers no votes as negative votes, using a threshold to determine the voting outcome. The threshold has to be set on a per-application basis because an optimal value for this threshold depends on the application. Each application and its user may have a different social contract, which defines the ways how users act. If a social contract defines a loosely-coupled collaboration with voluntary contribution, it may see fewer responses to voting requests than in a tightly-coupled collaboration, where contributions are compulsory. Thus, e.g., in a loosely-coupled online collaboration application the threshold has to be set to a lower value than in a tightly-coupled scientific collaboration application.

In contrast to $\mathrm{P} 2 \mathrm{P}$ recommendation systems, which are typically used for recommending complete documents, PeerVote is used for managing changes of a document in a decentralized collaboration application. PeerVote allows previous authors to review changes. However, new documents and early proposals are prone to malicious behavior because only few or none previous editors exist. A possible solution is to combine a recommendation system with PeerVote. A second issue is that authors can block changes forever, if they do not respond to a review request. If for a first change proposal the previous author does not respond, then a majority is never reached. Furthermore, for old documents, previous authors may not be as responsive as for newer documents. Thus, such a threshold needs to be time-based, where old documents need less votes for a change. A third issue is that the list of previous authors grows, resulting in a polynomial traffic. This could be solved by limiting the capacity of this list.

The voting mechanism is not Sybil attack 9] proof, since it does not prevent a user from acquiring multiple peer identities. Mechanisms to prevent or detect acquisition of multiple identities have to be implemented. Such mechanisms may be implemented with certificates binding peer identifiers to real-world identities, either with a trusted third part or with a web of trust. Another mechanism to limit multiple identities is by exchanging or paying with resources to participate or not be excluded [4].

Future work will investigate how the PeerVote mechanism can be combined with an incentive scheme. With such a combination, each vote can be traded 
to encourage peers to review changes or to store and provide documents. Further work will also investigate PeerVote in other application domains. In other application domains more voting choices could be offered to voters.

Acknowledgements. This work has been performed partially in the framework of the EU IST Project EC-GIN (FP6-2006-IST-045256) as well as the EU IST NoE EMANICS (FP6-2004-IST-026854). Many thanks go to Lennart Svensson, who did initial work on this topic in his Master's thesis [22.

\section{References}

1. Adler, T.B., de Alfaro, L.: A content-driven reputation system for the wikipedia. In: Proceedings of the 16th international conference on World Wide Web (WWW 2007), New York, NY, USA, pp. 261-270 (2007)

2. Bocek, T.: TomP2P - A Distributed Multi Map (2009), http://www.csg.uzh.ch/publica-tions/software/TomP2P

3. Bocek, T., Stiller, B.: Peer-to-Peer Large-scale Collaborative Storage Networks. In: Bandara, A.K., Burgess, M. (eds.) AIMS 2007. LNCS, vol. 4543, pp. 225-228. Springer, Heidelberg (2007)

4. Castro, M., Druschel, P., Ganesh, A., Rowstron, A., Wallach, D.S.: Secure routing for structured peer-to-peer overlay networks. SIGOPS Operating Systems Review 36(SI), 299-314 (2002)

5. Cetintemel, U., Keleher, P.J.: Light-Weight Currency Management Mechanisms in Deno. In: Proceedings of the 10th International Workshop on Research Issues in Data Engineering (RIDE), San Diego, CA, USA, February 2000, pp. 17-24 (2000)

6. Chaum, D.: Blind signature system. In: CRYPTO 1983: Advances in Cryptology, New York, USA, p. 153 (1983)

7. Cohen, B.: Incentives Build Robustness in BitTorrent. In: Proceedings of the 1st Workshop on Economics of Peer-to-Peer Systems (P2PECON), Berkeley, CA, USA (June 2003)

8. Dabek, F., Kaashoek, M.F., Karger, D., Morris, R., Stoica, I.: Wide-area cooperative storage with CFS. In: Proceedings of the 18th ACM Symposium on Operating Systems Principles (SOSP), Chateau Lake Louise, Ban (October 2001)

9. Douceur, J.R.: The sybil attack. In: Druschel, P., Kaashoek, M.F., Rowstron, A. (eds.) IPTPS 2002. LNCS, vol. 2429, pp. 251-260. Springer, Heidelberg (2002)

10. EMANICSLab, http://emanicslab.csg.uzh.ch/

11. Gifford, D.K.: Weighted Voting for Replicated Data. In: Proceedings of the 7th ACM Symposium on Operating Systems Principles (SOSP), pp. 150-162 (1979)

12. Gray, J.: Notes on data base operating systems. In: Flynn, M.J., Jones, A.K., Opderbeck, H., Randell, B., Wiehle, H.R., Gray, J.N., Lagally, K., Popek, G.J., Saltzer, J.H. (eds.) Operating Systems. LNCS, vol. 60, pp. 393-481. Springer, Heidelberg (1978)

13. Hardekopf, B., Kwiat, K., Upadhyaya: Secure and Fault-Tolerant Voting in Distributed Systems. In: Proceedings of the 2001 IEEE Aerospace Conference, Big Sky, Montana, USA, March 2001, vol. 3 (2001)

14. Korsgaard, T., Jensen, C.: Reengineering the Wikipedia for Reputation. In: Proceedings of the 4th International Workshop on Security and Trust Management (STM 2008), Trondheim, Norway, June 2008, pp. 71-84 (2008) 
15. Kubiatowicz, J., Bindel, D., Chen, Y., Eaton, P., Geels, D., Gummadi, R., Rhea, S., Weatherspoon, H., Weimer, W., Wells, C., Zhao, B.: OceanStore: An Architecture for Global-scale Persistent Storage. In: Proceedings of the 9th international Conference on Architectural Support for Programming Languages and Operating Systems (ASPLOS 2000), Cambridge, MA, USA (November 2000)

16. Maymounkov, P., Mazières, D.: Kademlia: A peer-to-peer information system based on the XOR metric. In: Druschel, P., Kaashoek, M.F., Rowstron, A. (eds.) IPTPS 2002. LNCS, vol. 2429, p. 53. Springer, Heidelberg (2002)

17. Morris, J.C., Lüer, C.: DistriWiki: A Distributed Peer-to-Peer Wiki. In: Proceedings of the 2007 International Symposium on Wikis (WikiSym 2007), Montreal, Quebec, Canada, October 2007, pp. 69-74 (2007)

18. Mukherjee, P., Leng, C., Schür, A.: Piki - A Peer-to-Peer based Wiki Engine. In: Proceedings of the 2008 8th International Conference on Peer-to-Peer Computing (P2P 2008), Washington, DC, USA, September 2008, pp. 185-186 (2008)

19. Rodrig, M., LaMarca, A.: Decentralized weighted voting for P2P data management. In: Proceedings of the 3rd ACM international workshop on Data engineering for wireless and mobile access (MobiDe 2003), San Diego, CA, USA, pp. 85-92 (2003)

20. Rowstron, A., Druschel, P.: Pastry: Scalable, distributed object location and routing for large-scale peer-to-peer systems. In: Guerraoui, R. (ed.) Middleware 2001. LNCS, vol. 2218, pp. 329-350. Springer, Heidelberg (2001)

21. Shamir, A.: How to Share a Secret. Communications of the ACM 22(11), 612-613 (1979)

22. Svensson, L.: Decentralized Secure and Incentive-compatible Votin in P2P Networks. Master's thesis, Communication Systems Group, IFI, University of Zurich, Switzerland (March 2007)

23. Urdaneta, G., Pierre, G., van Steen, M.: A Decentralized Wiki Engine for Collaborative Wikipedia Hosting. In: Proceedings of the 3rd International Conference on Web Information Systems and Technologies (WEBIST), Barcelona, Spain (March 2007)

24. Warner, B., Wilcox-O'Hearn, Z., Kinninmont, R.: Tahoe: A Secure Distributed Filesystem (March 2008), http://allmydata.org/ warner/pycon-tahoe.html

25. Weiss, S., Urso, P., Molli, P.: Wooki: A P2P Wiki-Based Collaborative Writing Tool. In: Benatallah, B., Casati, F., Georgakopoulos, D., Bartolini, C., Sadiq, W., Godart, C. (eds.) WISE 2007. LNCS, vol. 4831, pp. 503-512. Springer, Heidelberg (2007)

26. Wikipedia, http://www.wikipedia.org

27. Wuala, your files online, http://wua.la

28. Zattoo - TV meets PC, http://zattoo.com/ 